\title{
Design of a Web-Based Symptom Management Intervention for Cancer Patients
}

\author{
Christine M. Newlon ${ }^{1}$, Chin-Chun A. Hu ${ }^{1}$, Renee M. Stratton ${ }^{2}$, \\ and Anna M. McDaniel ${ }^{1,2}$ \\ ${ }^{1}$ Indiana University School of Informatics, 535 W. Michigan, Indianapolis, IN 46202 \\ ${ }^{2}$ Indiana University School of Nursing, 1111 Middle Drive NU 340, Indianapolis, IN 46202 \\ \{cnewlon, chinghu, rstratto, amcdanie\} @iupui.edu
}

\begin{abstract}
The discipline of Human-Computer Interaction design has potential for significant benefit to the field of health informatics. This paper describes the design approach used to develop a web-based interface to help cancer patients manage their chemotherapy side effects. Previous versions of this intervention utilizing telephone technology had been efficacious, but limited. The paper discusses the design decisions made in order to leverage the potential benefits of the Internet in supporting patients while avoiding the potential pitfalls that the patients may encounter with a web-based approach.
\end{abstract}

Keywords: Human-computer interaction, iterative design, cancer, web-based intervention, symptom management, evidence-based practice, reading level, continuous evaluation.

\section{Introduction}

Human-computer interaction (HCI), as a design philosophy, is an approach likely to yield significant benefits to the growing field of health informatics. This interactive design technique has increased our ability to develop new interventions and translate existing interventions, which can be either delivered within the health care system or extended through the Internet into people's homes. During an HCI design process, designers and users collaborate to identify the requirements of an application. The interface is then designed and implemented through an iterative process with feedback from the users at each stage [1]. Current conditions favor such application development in the field of healthcare. Behavioral scientists have already developed and tested efficacious interventions to support patients and families before, during, and long after their diagnosis $[2,3,4,5]$. The next essential step is to translate these interventions for patients and families struggling with disease, and ultimately for the general population, through the use of interaction design and web-based technology. This paper describes the human computer interaction design approach used to develop one such application, a web-based interface to help cancer patients manage their chemotherapy side effects. 


\subsection{A Challenge in Symptom Management}

Cancer is one of the most prevalent chronic illnesses in this country. Management of patient symptoms during chemotherapy is a challenge in clinical oncology, requiring a significant amount of time and effort to communicate with patients and families. Self-management of symptoms during cancer treatment can be a considerable burden that can negatively impact quality of life for cancer patients and their families. Therefore, an efficacious intervention to support symptom management has the potential to increase the efficiency and effectiveness of clinical care [6].

\subsection{Symptom Management Interventions}

The symptom management intervention described in this paper has grown through several iterations, each carefully studied following the principles of evidence-based practice. Initially this symptom management concept was implemented both through a series of phone calls by a nurse, and through an Automated Telephone Symptom Management (ATSM) system [6]. In this clinical trial, 435 patients were randomized to receive either automated calls or a similar intervention delivered over the telephone by trained cancer nurses. After reporting their symptoms over the phone, patients were referred to a paper-based "toolkit" of self care strategies for managing those symptoms that were reported to be above a pre-established threshold. Both arms produced significant reduction in symptoms over baseline while the ATSM intervention was more cost effective than the telephone-based nurse counseling [6]. However, the automated telephone system posed limitations, both from a technical stand point and from a usability perspective (Table 1.) Advances in web technology could offer potential solutions for these problems. The current project begins a new iteration, designed to translate the self-management intervention to a web-based platform.

Table 1. Limitations posed by ATSM system [6]

\begin{tabular}{|c|c|}
\hline Perspective & Limitations \\
\hline Technical & $\begin{array}{l}\text { - An increased number of phone lines were needed as patients were } \\
\text { added. }\end{array}$ \\
\hline Effectiveness & $\begin{array}{l}\text { - Patients were required to follow the pace of the system. } \\
\text { - There was no way to change incorrect responses. }\end{array}$ \\
\hline Efficiency & $\begin{array}{l}\text { - Option choices were repeated, which increased call time. } \\
\text { - Resumption of symptom reporting after interruptions was complicated. } \\
\text { - Patients were required to remember/write down a list of significant } \\
\text { symptoms and refer to a secondary source (toolkit) for self-care tips. }\end{array}$ \\
\hline Accessibility & $\begin{array}{l}\text { - Patients were required to be available at a predetermined time and } \\
\text { number. } \\
\text { - The contact method was limited to one patient-provided number. } \\
\text { - It was hard to carry around the paper-based toolkit, limiting access to } \\
\text { the symptom management tips. } \\
\text { - Symptom history/progress could be monitored only by the health care } \\
\text { providers. }\end{array}$ \\
\hline
\end{tabular}




\section{Background}

\subsection{Potential Uses of the Internet for Cancer Care}

The Internet offers a new paradigm for cancer care delivery. Cancer is one of the most prevalent chronic illnesses in this country. Scientific and medical advances in the detection, prevention, and treatment of cancers have resulted in a decreased incidence of new cancers and an overall decline in cancer death rates, thus increasing the number of people living with cancer. Survivors and their families have significant information needs concerning such things as diagnosis and treatment, management of symptoms, coordination of care, and prevention of further problems [7].

Web technology has the potential to dramatically improve the delivery of cancer care. The Internet can provide extensive information regarding treatment and diagnosis. Patients actively seek such information soon after the diagnosis of cancer and before starting treatment [8]. They typically want to know as much information as possible, and may not be satisfied with the amount of information from health care professionals [8]. Therefore, cancer patients and their relatives turn to the Internet as an alternative information source [9]. Their reasons for using the Internet include finding information quickly and easily, sharing their experiences with others, and obtaining health-related information confidentially $[9,10]$.

Research has demonstrated that increased knowledge of cancer enhances coping in cancer patients [11]. Newly diagnosed cancer patients who access the Internet for health information report feeling empowered to make decisions about their health and the management of their disease [12]. Patients report higher levels of satisfaction with health information obtained on the Internet compared to that from other media such as television, newspaper, magazine, and radio [8].

Therefore, it follows that one potential application of web technology in cancer care is through its use to monitor symptoms and deliver interventions that can improve the quality of life for cancer patients and their families. Cancer patients experience a number of life altering symptoms during treatment. Management of chemotherapy-related symptoms is a significant burden to cancer patients and their families. Information about strategies to reduce symptoms can be complex and difficult to manage. The Internet can be an important source of information and support for symptom management $[9,10,13]$. Additionally, there is a strong positive relationship between Internet use and cancer patients' self-efficacy for participation in care [11].

\subsection{Issues of Concern in Internet-Based Intervention}

Although web-based sources of cancer-related information and support are increasingly common and have been shown to have a positive impact on patient outcomes $[8,14]$, there are important issues to consider in designing a cancer support application. First, even though most patients prefer to have as much information as possible to inform their health care decision making, some cancer patients have reported being overwhelmed with too much information [8, 15]. In addition, much of the available information on the Internet is not evidence-based [6] and may be inaccurate or misleading [16]. Unfortunately, little of web-based cancer information is 
peer reviewed, and few sites differentiate information according to source, quality, or accuracy.

A second issue is the readability of cancer information. Currently, most online information is at a high school (Grade 11-12) or collegiate level (Grade 13+), which is much higher than the average reading level (Grade 8) [16]. An inappropriate reading level could be a barrier to accessing the health information on the Internet or in print. Web-based multimedia technology allows information to be presented in a variety of formats (e.g., audio, and video), independent of reading ability. Furthermore, the Internet has the potential to facilitate the delivery of customized information, which has been shown to increase responsiveness to health messages and may lead to more positive behavior changes [17].

\section{A Web-Based Symptom Management Toolkit}

It is against this background of both potential and concern that we have undertaken the next iteration of symptom management intervention. Our proposed solution to address the limitations of the ATSM intervention is a secure Symptom Management Toolkit deployed on a web-based platform, which includes a secure server to host the application, a database for content storage, Flash programming to present the interface, algorithms to control sequencing of events, and an authentication system for user identification. Using the Internet for cancer care delivery not only makes possible powerful functions that were previously unavailable with other technology, but also allows patients to access information and perform their symptom management activities anytime and anywhere convenient for them. We describe below how we addressed the potential issues of concern through our design.

\subsection{Cancer Patients as the Center of Our Design}

It was our intention to develop an easy-to-follow system with helpful functionalities one that could fully utilize technology to support cancer patients' symptom management effort and increase their awareness of their health condition. To this end, user characteristic variances such as reading level, computer literacy, and cognitive ability, were considered in the design. We also identified and addressed three central user needs: help with symptom management, improved patient-provider communication, and increased coordination of care. The system was intended to be used by patients outside the clinic setting. Therefore, the system's operation is intuitive, requiring a minimum amount of patient learning (e.g., clicking the next button will advance system to the next screen.) Since the system will be used by patients in a real world context, interruptions while performing tasks are possible. The design allows patients to easily resume system activity without having to start from the beginning.

\subsection{System Functionality}

The system was iteratively designed, beginning with a series of meetings with cancer survivors and health care providers to assess user needs and identify requirements, followed by usability testing of the system prototype to evaluate and improve the 
design. Functional requirements elicited through this process were translated into interface components. In addition, we designed a feedback component that integrates continuous evaluation into the normal operation of the system.

Symptom Assessment. The system begins each weekly session by sending patients an automated symptom assessment request with an embedded link to the login screen. (If a patient fails to $\log$ into the system within 24 hours, the system sends a reminder message.) After logging in, patients are required to assess 9 symptoms commonly experienced by people undergoing chemotherapy (Fig. 1). Through the instructing and conversing interaction modes, patients proceed at their own pace to report symptoms they have experienced, rating the severity of each symptom, and the extent to which it interfered with their enjoyment of life, relationship with others, daily activity, and mood. The assessment is controlled in a linear fashion to ensure data integrity. Then, patients have the opportunity to report more symptoms by selecting from a secondary list of symptoms. Input is progressively saved into the database. Patients can stop at anytime and easily resume from where it was left off the next time they login the system. After assessing their symptoms, patients are presented a Symptom Summary (a bar graph summary of the data entered) from which they can review and edit information they have entered. [NOTE: Patients are instructed to call their oncologist if they are experiencing severe symptoms or have a medical concern.]

Custom Toolkit. Upon assessment completion, the system presents patients with a Custom Toolkit containing self-care strategies for symptoms assessed at a level of 4 or higher out of 10 (Fig. 2). This threshold was derived from multiple studies using the Symptom Experience Inventory items in clinical practice [18]. Content in the toolkit is quality-filtered, evidence-based cancer information. It is written at a $5^{\text {th }}$ grade reading level to support readability. For patients wanting to increase the font size, a text-resizing function is offered. In addition, a print function is offered allowing a printable, text-only version of the symptom management information. [NOTE: Patients may also access information on any of the symptoms from the Full Toolkit, even if they are not experiencing those symptoms at the time.]

Symptom History. Web technology has made it possible to retrieve and display symptom assessment data in a visualized format, allowing both patients and health care providers to easily monitor changes in symptoms over time. Graphical representations of the symptom history are presented in order of the highest to lowest severity rating for the latest assessment to help with decisions on which symptoms to attend to first (Fig. 3).

Provider Alerts. Another function of the system is to immediately notify providers by e-mail when severe symptoms have been reported (rated 7 or higher out of 10). (Since e-mail transmissions are not considered HIPAA compliant, no patient names are included in the e-mail notification.) After receiving an email alert and logging in, providers can review the symptom history of the patient with severe symptoms to determine follow-up actions. They also have the option of printing the report for further follow-up as would be part of their clinical practice patterns when addressing a patient concern. 


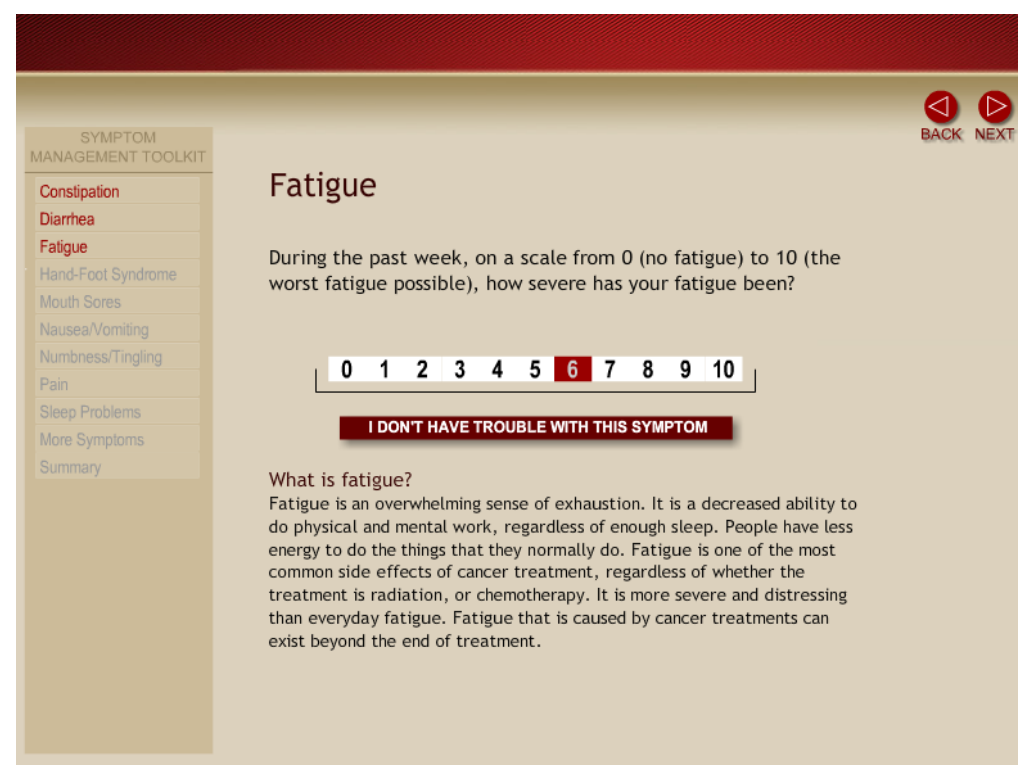

Fig. 1. Symptom Assessment

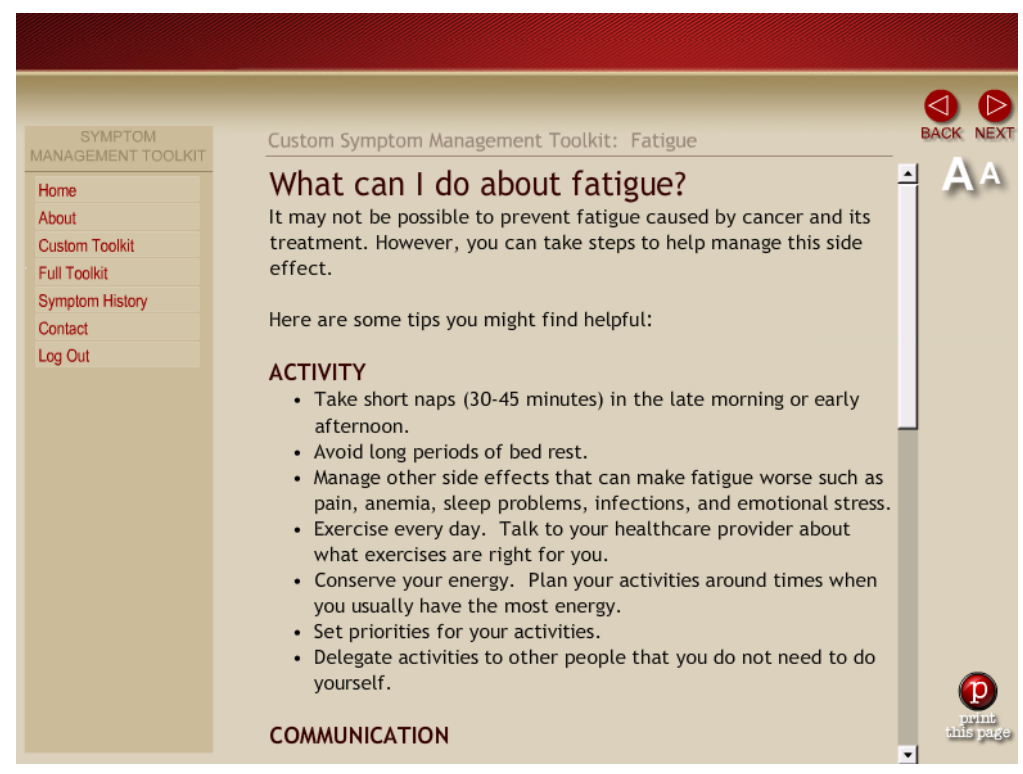

Fig. 2. Custom Toolkit

Continuous Evaluation. Two days after the assessment request, another automated email message is sent asking patients to update the current status of their significant symptoms (those rated at 4 or higher, on a scale of 0-10 in the latest assessment.) This evaluation function allows patients to report symptom status in a three option 
response format (i.e., worse, about the same, or better) using a radio button interface. This design is intended to emulate a follow-up encounter with a health care provider who is monitoring symptom severity. In addition to the symptom status update, the evaluation function uses this opportunity to gather information from the patients about the utility of the toolkit, and its ease of use. This stream of information will provide the system's designers with the continuous feedback they need to monitor the effectiveness of the toolkit in meeting patient needs.

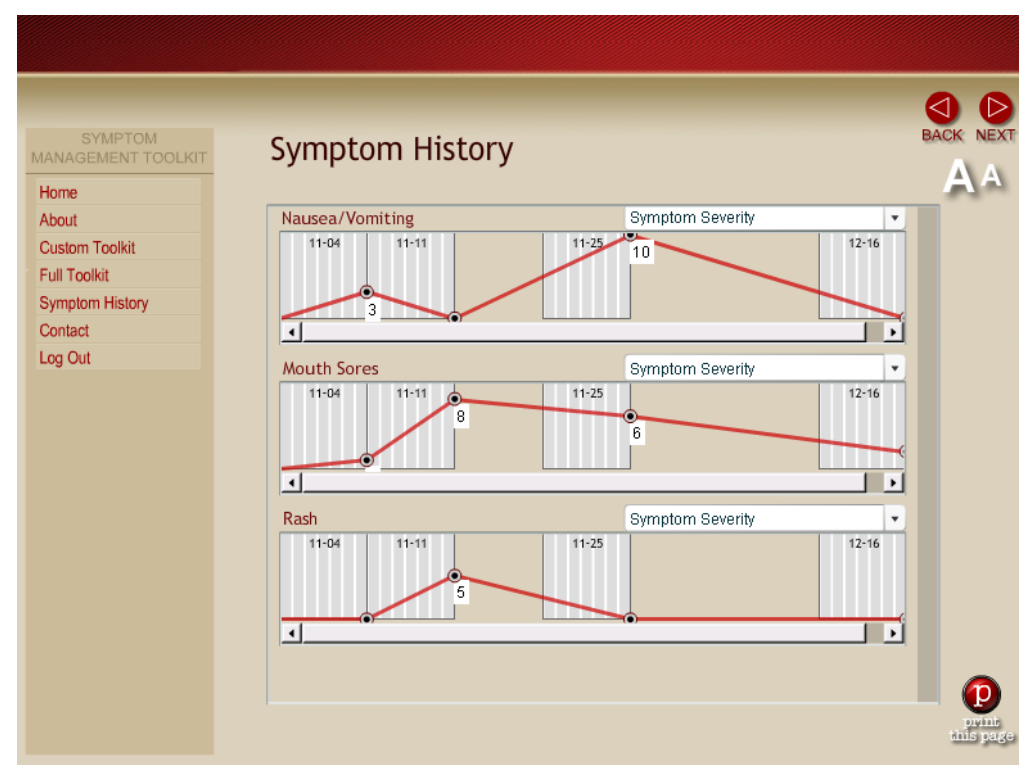

Fig. 3. Symptom History

\section{Conclusions}

Since the web system essentially serves as a platform for the "translation" of the previously studied ATSM intervention, and that intervention has been shown to be efficacious in a randomized clinical trial [6], the results of that study support our use of technology-driven cancer control interventions. In addition, a recent study by Basch and colleagues showed that online reporting of toxicity during chemotherapy is feasible and is acceptable to cancer patients [19], lending further support for our approach. However, the success of the system will still depend on how successful we have been at avoiding the potential pitfalls of web design. Even with the tremendous potential of the Internet, the process of designing the human-computer interaction still depends on iteration and testing.

\subsection{Insights from the Design Process}

While our usability test results were good overall, with a slight upward trend as the site was improved, it was instructive to see the results on specific features. For 
instance, we discovered we needed a button saying, "I do not have this symptom." Otherwise, several of our testers felt obligated to enter a non-zero number for every possible symptom. For them, not having a symptom might be rated as a " 2 " on our " $0-10 "$ scale. This sort of insight is impossible to achieve without having actual users test the application.

\subsection{Next Steps}

As this paper went to press, we launched a full pilot study to test the feasibility of this web-based symptom monitoring and management system. In this pilot study, the system is being put in use by real patients and in real context. During the eight week study, patients will assess their symptoms and evaluate the system once a week. Throughout the duration, patients will receive customized information that may help relieve their chemotherapy-related symptoms. Their providers will monitor symptom severities, and may further customize treatment based on assessments. From the results, we hope to learn if the customized information was presented in a usable format, whether the customized information was used effectively to manage symptoms, and overall patient satisfaction with the system. This will allow us to validate the new system against our previous interventions.

System enhancements are planned based on responses from the usability test follow-up interviews and the pilot study results. For instance, the responses indicate that a customizable reminder sheet is desired that users can print and bring to a doctor's appointment. In future iterations, a caregiver application is also planned that will provide support for family members of cancer patients.

\subsection{Transcending Usability-Based Approaches}

For the first version of the web-based Symptom Management Toolkit, our primary focus was usability, and only the most critical functions, supported by minimum visual elements, were incorporated into the system's design. Noting the increased importance of human factors in product creation, we plan to go beyond traditional usability-based approaches in the next version and explore affective approaches to understand cancer patients holistically. In addition to cognitive and physical abilities, we will consider all of the possible hedonic, emotional, and practical benefits offered by this system by further investigating the role it plays in patients' everyday and working lives [20]. Ultimately, our goal is to design a system that is not only usable but also provides comfort to cancer patients.

Acknowledgements. The authors would like to thank Dr. Anthony Faiola at the Indiana University School of Informatics, the development team, Robert S. Comer and Shielly Hartanto, and content experts, Drs. Charles W. and Barbara Given. The project is the result of collaboration between the IU Melvin and Bren Simon Cancer Center, Walther Cancer Foundation, and Indiana University Schools of Nursing, Informatics, and Medicine. 


\section{References}

1. Helander, M. (ed.): Handbook of human-computer interaction. Elsevier, New York (1997)

2. Kurtz, M.E., Kurtz, J.C., Given, C.W., Given, B.: Symptom cluster among cancer patients and effects of a cognitive behavioral intervention. Cancer Therapy 5, 105-112 (2007)

3. Sherwood, P., Given, B.A., Given, C.W., Champion, V.L., Doorenbos, A.Z., Azzouz, F., Kozachik, S., Wagler-Ziner, K., Monahan, P.O.: A cognitive behavioral intervention for symptom management in patients with advanced cancer. Oncol. Nurs. Forum. 32(6), 1190-1198 (2005) [Online]

4. Rawl, S., Given, B., Given, C.W., Champion, V., Kozachik, S., Barton, D.: Interventions to improve psychological functioning for newly diagnosed patients with cancer. Oncol. Nurs. Forum. 29(6), 967-975 (2002)

5. Given, C.W., Given, B., Rahbar, M., Jeon, S., McCorkle, R., Cimprich, B., et al.: Effect of a cognitive behavioral intervention on reducing symptom severity during chemotherapy. $\mathrm{J}$. Clin. Oncol. 22(3), 507-516 (2004)

6. Sikorskii, A., Given, C.W., Given, B., Jeon, S., Decker, V., Decker, D., Champion, V., McCorkle, R.: Symptom management for cancer patients: a trial comparing two multimodal interventions. J. Pain Symptom Manage. 34(3), 253-264 (2007)

7. Hewitt, M., Grennfield, S., Stoval, E. (eds.): From cancer patient to cancer survivor: Lost in Transition. National Academies Press, Washington (2006)

8. Eysenbach, G.: The impact of the Internet on cancer outcomes. CA Cancer J. Clin. 53(6), 356-371 (2003)

9. National Cancer Institute. About Cancer Survivorship Research: Survivorship Definitions, http://dccps.nci.nih.gov/ocs/definitions.html

10. Satterlund, M.J., McCaul, K.D., Sandgren, A.K.: Information gathering over time by breast cancer patients. J. Med. Internet Res. 5(3), e15 (2003)

11. Kyngas, H., Mikkonen, R., Nousiainen, E.M., Rytilahti, M., Seppänen, P., Vaattovaara, R., Jämsä, T.: Coping with the onset of cancer: coping strategies and resources of young people with cancer. Eur. J. Cancer Care. 10(1), 6-11 (2001)

12. Bass, S.B., Ruzek, S.B., Gordon, T.F., Fleisher, L., McKeown-Conn, N., Moore, D.: Relationship of Internet health information use with patient behavior and self-efficacy: experiences of newly diagnosed cancer patients who contact the National Cancer Institute's Cancer Information Service. J. Health Commun. 11(2), 219-236 (2006)

13. Raupach, J.C., Hiller, J.E.: Information and support for women following the primary treatment of breast cancer. Health Expect. 5(4), 289-301 (2002)

14. Mills, M.E., Sullivan, K.: The importance of information giving for patients newly diagnosed with cancer: a review of the literature. J. Clin. Nurs. 8(6), 631-642 (1999)

15. Leydon, G.M., Boulton, M., Moynihan, C., Jones, A., Mossman, J., Boudioni, M., McPherson, K.: Cancer patients' information needs and information seeking behaviour: in depth interview study. BMJ 320(7239), 909-913 (2000)

16. Friedman, D.B., Hoffman-Goetz, L., Arocha, J.F.: Health literacy and the world wide web: Comparing the readability of leading incident cancers on the Internet. Med. Inform. Internet in Med. 31(1), 67-87 (2006)

17. Kreuter, M.W., Strecher, V.J., Glassman, B.: One size does not fit all: the case for tailoring print materials. Ann. Behav. Med. 21(4), 276-283 (1999) 
18. Kurtz, M.E., Kurtz, J.C., Given, C.W., Given, B.: Effects of a symptom control intervention on utilization of health care services among cancer patients. Med. Sci. Monit. 12(7), CR319-24 (2006)

19. Basch, E., Artz, D., Dulko, D., Scher, K., Sabbatini, P., Hensley, M., Mitra, N., Speakman, J., McCabe, M., Schrag, D.: Patient online self-reporting of toxicity symptoms during chemotherapy. J. Clin. Oncol. 23(15), 3552-3561 (2005)

20. Jordan, P.W.: Designing pleasurable products. Taylor \& Francis, London (2000) 